\title{
Apnoeic Oxygenation During One Lung Ventilation - a Case Report
}

\author{
Spencer, L; Simões, V.; Oliveira, C.; Marques, C., Salta, C. Poeira, R.
} Central Lisbon Hospitalar Center, Portugal - Head of department: Isabel Fragata, MD

\section{INTRODUCTION}

Hypoxemia is a possible complication of switching from two-lung to one-lung ventilation (OLV), as shunt fraction increases and oxygenation is impaired. Factors that can contribute to and/or exacerbate hypoxemia include previous lung disease and intra-operative positioning.

Hypoxemia during OLV may be prevented using a ventilation strategy that avoid alveolar collapse while minimally impairing the dependent lung perfusion. When other causes are excluded such as malposition or obstruction of the double-lumen tube, hypoxemia can be improved by (1) increasing oxygen inspired fraction, (2) modifying ventilatory patterns or (3) using strategies to oxygenate the non-ventilated lung.

\section{CASE REPORT}

- 86 year-old female

- Diagnosis: Spontaneous left haemothorax

- Proposed surgery: Drainage and clot remotion by thoracotomy

- Past history: Diabetes Mellitus type 2, hypertension, atrial fibrillation under oral anticoagulation with warfarine and recent pneumoniae in the right lung

- No past surgical or anesthetic history

\section{Preoperative evaluation}

- Laboratory evaluation: anemia (hemoglobin $6.9 \mathrm{~g} / \mathrm{dL}$ ), hyperlactacidemia $(6.6 \mathrm{mg} / \mathrm{dL})$ and hyponatremia $(125 \mathrm{mEq} / \mathrm{L})$

- Pulmonary auscultation: absence of breath sounds on the left haemithorax base

- Cardiac auscultation: hypophonetic cardiac sounds

- Mild hypotension responsive to fluid therapy

\section{INTRA AND POSTOPERATIVE EVALUATION}

- Patient underwent urgent thoracotomy under general anesthesia.

- The pulse oximetry values were normal (98\%) until pulmonary exclusion be performed, after what hypoxemia ensued (83$87 \%)$, unresponsive to high inspiratory oxygen fraction $\left(\mathrm{FiO}_{2}\right)$ and to modification of the ventilator patterns.

- An open valve Waters system at $5 \mathrm{~L} / \mathrm{min}$ oxygen was then connected to the non-ventilated lung in order to promote passive oxygen diffusion. Oximetry values increased to $95 \%$ without significant surgery field impairment .

- Patient was transferred to the ICU, respiratory and hemodynamically stable, but died 6 days later.

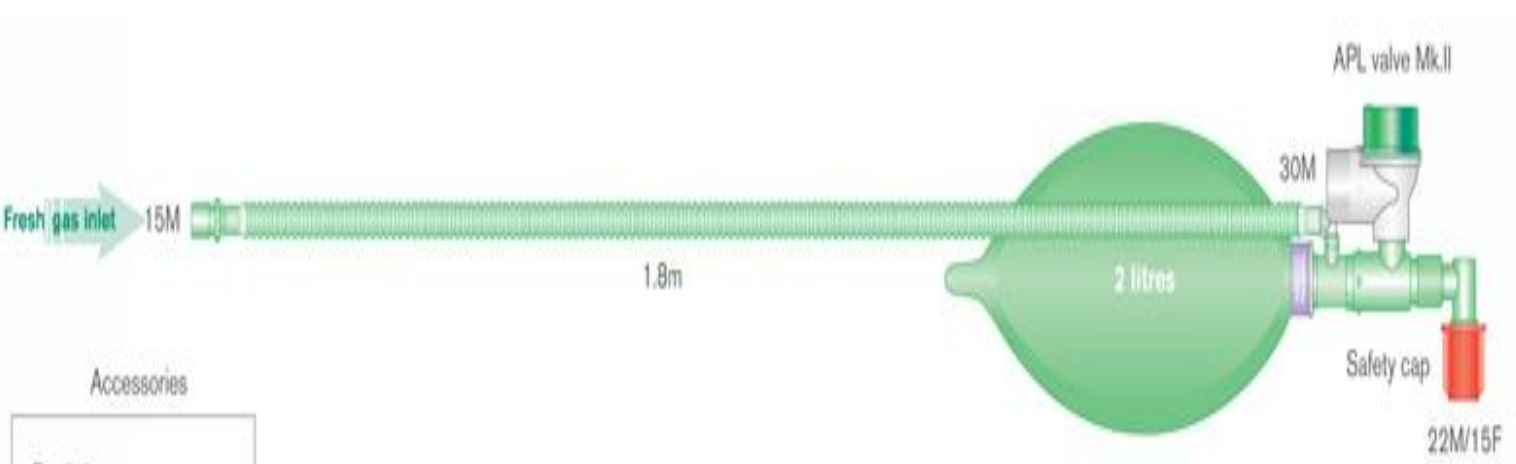

Picture 1: Mappleson C anesthetic system

\section{Discussion}

Apnoeic oxygenation is often used in the context of difficult airway management, following pre-oxygenation with high oxygen concentrations via nasal cannula, to prevent dessaturation. It generates a gas flow from the pharynx to the alveoli and therefore creates a positive alveolar oxygen gradient which leads to passive oxygen diffusion.

Hypoxemia management during one-lung ventilation require immediate intervention that includes: (1) confirming the position and patency of the tube; (2) increasing $\mathrm{FiO}_{2}$ to 100\%; (3) applying recruitment maneuvers and optimizing positive end-expiratory pressure (PEEP) on the ventilated lung and (4) ensuring adequate cardiac output and $\mathrm{O}_{2}$ carrying.

The last resource is two-lung ventilation or the pulmonary artery clamping but, before this, one can try to apply CPAP or blow-by oxygen to the surgical lung, passively improving oxygenation, but with increased risk of surgical field impairment.

In this case it was performed apnoeic oxygenation using a Waters circuit (Picture 1) with opened valve to prevent pulmonary inflation with good results. It proved to be a simple and efficient method for oxygen administration during OLV when other strategies have failed.

\section{CONCLUSION}

Hypoxemia is a possible complication during one-lung ventilation that can be improved through several strategies. This case report supports the role of apnoeic oxygenation as an effective and simple way to treat hypoxemia without impairment of the surgical field. 